\title{
Phase Behavior of Binary Mixture Systems of Two Crystallizable Components. Semicrystalline Polymers and Wholly-Crystalline Substances
}

\author{
Matsuo HiRami ${ }^{\dagger}$ and Tsunetoshi Matsuda \\ Research and Development Center, Unitika Ltd., \\ 23 Kozakura, Uji, Kyoto 611-0021, Japan \\ (Received January 18, 1999)
}

\begin{abstract}
The phase behavior of binary mixture systems of two crystallizable components including semicrystalline polymers and wholly-crystalline substances are investigated systematically. On the basis of the general melting temperature equation, the features of melting temperature diagram, i.e., higher and lower melting temperatures composition relations are presented for three categories of binary mixtures; (A) two wholly-crystalline substances, (B) a semicrystalline polymer and a wholly-crystalline substance and (C) two semicrystalline polymers. Applications to two cases of binary mixtures are demonstrated; first, in category B, mixtures of polyethylene and $n$-alkane (literature data of Mandelkern et al.), and second, in category $\mathrm{C}$, mixtures of nylon 6 and nylon 66 (our data in this series).
\end{abstract}

KEY WORDS Binary Mixtures / Melting Temperature Equation / Melting Temperature Diagram /

Phase Equilibrium / Semicrystalline Polymer / Wholly-Crystalline Substance /

The basis for understanding of the melting behavior of binary mixture systems involving a crystallizable polymer component was well established. ${ }^{1-3}$ In general, binary mixtures are composed of various substances with different types of crystalline characteristics, i.e., whollycrystalline, semicrystalline and non-crystalline (liquid). Binary mixtures of crystallizable polymers and liquid diluents have been studied most extensively. ${ }^{4}$ The relationship of melting temperature depression of a crystallizable polymer caused by a monomeric diluent was derived early by Flory $^{1}$ and later the similar relationship for mixtures of a crystalline-amorphous polymer pair was presented by Nishi and Wang. ${ }^{5}$ For binary mixtures where both components crystallize separately, two melting temperatures are usually observed. In this case, both of higher and lower melting temperaturecomposition relations have to be investigated. We wish to study the features of melting temperature diagram for binary mixture systems of two crystallizable components including semicrystalline polymers and wholly crystalline substances. From the general aspect, such binary mixture systems can be divided into three categories, i.e., (A) two wholly-crystalline substances, (B) combination of a semicrystalline polymer and a whollycrystalline substance and (C) two semicrystalline polymers.

In this work, we investigate the phase behavior of these binary mixture systems systematically. First, we derive the general melting temperature equation for binary mixture systems of two crystallizable components, from which higher and lower melting temperature-composition relations in the respective three categories can be deduced. Attention is directed to whether or not the Gibbs phase rule holds in these relations.

Applications to several cases of interest are presented; one is the analysis of literature data for binary mixtures of polyethylene and normal alkane reported by Mandelkern et al. $^{6}$ and the other is data for binary mixtures of nylon 6 and nylon 66 obtained in our

\footnotetext{
† To whom all correspondence should be addressed.
}

laboratory. The former corresponds to the category B and the latter the category $\mathrm{C}$.

\section{GENERAL MELTING TEMPERATURE EQUATION}

We consider a binary mixture system consisted of two crystallizable polymer components 1 and 2 . The condition of equilibrium between the respective crystalline phases and the surrounding amorphous (liquid) phase is represented as follows:

$$
\mu_{\mathrm{u} 1}^{\mathrm{c}}-\mu_{\mathrm{u} 1}^{\mathrm{o}}=\mu_{\mathrm{u} 1}-\mu_{\mathrm{u} 1}^{\mathrm{o}}
$$

and

$$
\mu_{\mathrm{u} 2}^{\mathrm{c}}-\mu_{\mathrm{u} 2}^{\mathrm{o}}=\mu_{u 2}-\mu_{u 2}^{\mathrm{o}}
$$

where $\mu_{\mathrm{u} 1}^{\mathrm{c}}$ and $\mu_{\mathrm{u} 2}^{\mathrm{c}}$ are the chemical potentials of the polymer repeating unit in the two crystalline phases, $\mu_{\mathrm{u} 1}$ and $\mu_{\mathrm{u} 2}$ are the chemical potentials of the unit in the surrounding liquid phase, and $\mu_{\mathrm{u} 1}^{\mathrm{o}}$ and $\mu_{\mathrm{u} 2}^{\mathrm{o}}$ are those of the unit in the standard states (pure liquid polymers) for the respective components 1 and 2. Since the left-hand sides of eq 1 and 2 correspond to the negative of the free energies of fusion per polymer unit of the components 1 and 2, they can be written

$$
\mu_{\mathrm{u} 1}^{\mathrm{c}}-\mu_{\mathrm{u} 1}^{\mathrm{o}}=-\Delta H_{\mathrm{u} 1}\left(1-T / T_{\mathrm{m} 1}^{\mathrm{o}}\right)
$$

and

$$
\mu_{\mathrm{u} 2}^{\mathrm{c}}-\mu_{\mathrm{u} 2}^{\mathrm{o}}=-\Delta H_{\mathrm{u} 2}\left(1-T / T_{\mathrm{m} 2}^{\mathrm{o}}\right)
$$

where $\Delta H_{\mathrm{u} 1}$ and $\Delta H_{\mathrm{u} 2}$ are the heats of fusion per repeating unit, and $T_{\mathrm{m} 1}^{\mathrm{o}}$ and $T_{\mathrm{m} 2}^{\mathrm{o}}$ are the melting points (in degrees Kelvin) of the pure polymers of the components 1 and 2 .

Expressions for the right-hand sides of eq 1 and 2 can be deduced from the mixing free energy of the Flory-Huggins theory. Let $\phi_{1}$ and $\phi_{2}\left(=1-\phi_{1}\right)$ be the volume fractions of the components 1 and 2 . In this work, the crystalline-amorphous proportions for the respective components should be incorporated in the framework of general treatment. For this purpose, we 
introduce the effective volume fractions and $\phi_{1}^{\prime}$ and $\phi_{2}^{\prime}$ $\left(=1-\phi_{1}^{\prime}\right)$ for the components 1 and 2 , which are related to the nominal volume fractions $\phi_{1}$ and $\phi_{2}$ by the definition of

$$
\phi_{1}^{\prime}=\phi_{1} \lambda_{1} /\left(\phi_{1} \lambda_{1}+\phi_{2} \lambda_{2}\right)
$$

and

$$
\phi_{2}^{\prime}=\phi_{2} \lambda_{2} /\left(\phi_{1} \lambda_{1}+\phi_{2} \lambda_{2}\right)
$$

where $\lambda_{1}$ and $\lambda_{2}$ are the fractions of the polymer in the amorphous, or liquid state for the components 1 and 2 . Then the right-hand sides of eq 1 and 2 are written

$$
\begin{aligned}
\mu_{\mathrm{u} 1}-\mu_{\mathrm{u} 1}^{\mathrm{o}}= & R T\left\{\left(\frac{1}{x_{1}}\right) \ln \left(\frac{\phi_{1} \lambda_{1}}{\phi_{1} \lambda_{1}+\phi_{2} \lambda_{2}}\right)\right. \\
& +\left[\frac{1}{x_{1}}-\left(\frac{z_{1}}{z_{2}}\right)\left(\frac{1}{x_{2}}\right)\right]\left(\frac{\phi_{2} \lambda_{2}}{\phi_{1} \lambda_{1}+\phi_{2} \lambda_{2}}\right) \\
& \left.+z_{1} \chi\left(\frac{\phi_{2} \lambda_{2}}{\phi_{1} \lambda_{1}+\phi_{2} \lambda_{2}}\right)^{2}\right\}
\end{aligned}
$$

and

$$
\begin{aligned}
\mu_{\mathrm{u} 2}-\mu_{\mathrm{u} 2}^{\mathrm{o}}= & R T\left\{\left(\frac{1}{x_{2}}\right) \ln \left(\frac{\phi_{2} \lambda_{2}}{\phi_{1} \lambda_{1}+\phi_{2} \lambda_{2}}\right)\right. \\
& +\left[\frac{1}{x_{2}}-\left(\frac{z_{2}}{z_{1}}\right)\left(\frac{1}{x_{1}}\right)\right]\left(\frac{\phi_{1} \lambda_{1}}{\phi_{1} \lambda_{1}+\phi_{2} \lambda_{2}}\right) \\
& \left.+z_{2} \chi\left(\frac{\phi_{1} \lambda_{1}}{\phi_{1} \lambda_{1}+\phi_{2} \lambda_{2}}\right)^{2}\right\}
\end{aligned}
$$

where $x_{1}$ and $x_{2}$ are the numbers of repeating units per molecule, $z_{1}$ and $z_{2}$ are the numbers of segments per structural unit for the respective components, and $\chi$ is the polymer-polymer interaction parameter. Note that $\chi$ is related to the originally defined Flory-Huggins interaction parameter $\chi_{12}$ by $\chi=\chi_{12} / z_{1} x_{1}{ }^{7,8}$

The general melting temperature equation for the components 1 and 2 can be obtained by substituting eq 3 and 7 into eq 1 , and eq 4 and 8 into eq 2 , respectively

$$
\begin{aligned}
\frac{1}{T}-\frac{1}{T_{\mathrm{m} 1}^{\mathrm{o}}}=- & \left(\frac{R}{\Delta H_{\mathrm{u} 1}}\right)\left\{\left(\frac{1}{x_{1}}\right) \ln \left(\frac{\phi_{1} \lambda_{1}}{\phi_{1} \lambda_{1}+\phi_{2} \lambda_{2}}\right)\right. \\
& +\left[\frac{1}{x_{1}}-\left(\frac{z_{1}}{z_{2}}\right)\left(\frac{1}{x_{2}}\right)\right]\left(\frac{\phi_{2} \lambda_{2}}{\phi_{1} \lambda_{1}+\phi_{2} \lambda_{2}}\right) \\
& \left.+z_{1} \chi\left(\frac{\phi_{2} \lambda_{2}}{\phi_{1} \lambda_{1}+\phi_{2} \lambda_{2}}\right)^{2}\right\}
\end{aligned}
$$

and

$$
\begin{aligned}
\frac{1}{T}-\frac{1}{T_{\mathrm{m} 2}^{\mathrm{o}}}=- & \left(\frac{R}{\Delta H_{\mathrm{u} 2}}\right)\left\{\left(\frac{1}{x_{2}}\right) \ln \left(\frac{\phi_{2} \lambda_{2}}{\phi_{1} \lambda_{1}+\phi_{2} \lambda_{2}}\right)\right. \\
+ & {\left[\frac{1}{x_{2}}-\left(\frac{z_{2}}{z_{1}}\right)\left(\frac{1}{x_{1}}\right)\right]\left(\frac{\phi_{1} \lambda_{1}}{\phi_{1} \lambda_{1}+\phi_{2} \lambda_{2}}\right) } \\
& \left.+z_{2} \chi\left(\frac{\phi_{1} \lambda_{1}}{\phi_{1} \lambda_{1}+\phi_{2} \lambda_{2}}\right)^{2}\right\}
\end{aligned}
$$

\section{APPLICATIONS}

\section{Mixtures of Two Wholly-Crystalline Substances}

Two Chemically Identical Species with Different Molecular Weights. In this case, two melting temperature relationships, $T=T_{\mathrm{m} 1}$ and $T=T_{\mathrm{m} 2}$ for the components 1 and 2 can be obtained from eq 9 and 10 , respectively, by putting $\lambda_{1}=\lambda_{2}=1,\left(z_{1} / z_{2}\right)=1, \Delta H_{\mathrm{u} 1}=$ $\Delta H_{\mathrm{u} 2} \equiv \Delta H_{\mathrm{u}}$ and $\chi=0$, namely we have

$$
\frac{1}{T_{\mathrm{m} 1}}-\frac{1}{T_{\mathrm{m} 1}^{\mathrm{o}}}=-\left(\frac{R}{\Delta H_{\mathrm{u}}}\right)\left[\frac{\ln \left(1-\phi_{2}\right)}{x_{1}}+\left(\frac{1}{x_{1}}-\frac{1}{x_{2}}\right) \phi_{2}\right]
$$

and

$$
\frac{1}{T_{\mathrm{m} 2}}-\frac{1}{T_{\mathrm{m} 2}^{\mathrm{o}}}=-\left(\frac{R}{\Delta H_{\mathrm{u}}}\right)\left[\frac{\ln \phi_{2}}{x_{2}}+\left(\frac{1}{x_{2}}-\frac{1}{x_{1}}\right)\left(1-\phi_{2}\right)\right]
$$

If we introduce $\bar{x}$, defined by

$$
\frac{1}{\bar{x}}=\frac{1-\phi_{2}}{x_{1}}+\frac{\phi_{1}}{x_{2}}
$$

then, eq 11 and 12 are reduced to

$$
\frac{1}{T_{\mathrm{m}}}-\frac{1}{T_{\mathrm{m}}^{\mathrm{o}}}=-\left(\frac{R}{\Delta H_{\mathrm{u}}}\right)\left(\frac{\ln \phi}{x}+\frac{1}{x}-\frac{1}{\bar{x}}\right)
$$

Equation 14 was derived by Mandelkern ${ }^{6}$ using a somewhat different procedure as the melting temperature relationship for mixtures of chemically identical species that differ in molecular weight. As an interesting application, the phase diagram for binary mixtures of two $n$-alkanes with different molecular weights, e.g., $\mathrm{C}_{40} \mathrm{H}_{82}$ and $\mathrm{C}_{60} \mathrm{H}_{122}$ can be constructed with the use of the combination of eq 11 and eq 12 .

Two Low Molecular Weight Crystalline Substances. In case of binary mixtures of two low molecular weight crystalline substances, the volume fractions $\phi_{1}$ and $\phi_{2}$ in eq 7 and 8 can be replaced by the number mole fractions $N_{1}$ and $N_{2}$ From eq 9 and 10, by putting $\lambda_{1}=\lambda_{2}=1, z_{1} / z_{2}=1, \chi=0$ and in the limit as $x_{1}=x_{2} \rightarrow 1$, at $T=T_{\mathrm{m} 1}$ and $T=T_{\mathrm{m} 2}$ for the components 1 and 2 , we have

$$
\frac{1}{T_{\mathrm{m} 1}}-\frac{1}{T_{\mathrm{m} 1}^{\mathrm{o}}}=-\left(\frac{R}{\Delta H_{1}}\right) \ln N_{1}
$$

and

$$
\frac{1}{T_{\mathrm{m} 2}}-\frac{1}{T_{\mathrm{m} 2}^{\mathrm{o}}}=-\left(\frac{R}{\Delta H_{2}}\right) \ln N_{2}
$$

where $\Delta H_{1}$ and $\Delta H_{2}$ are the heats of fusion for the components 1 and 2. Equations 15 and 16 are the familiar melting point relationships for the classical ideal solution.

\section{Mixtures of a Semicrystalline Polymer and a Wholly- Crystalline Substance}

Two Chemically Identical Species with Different Molecular Weights. In this case, a semicrystalline polymer shall be designated as the component 2 and a wholly- 

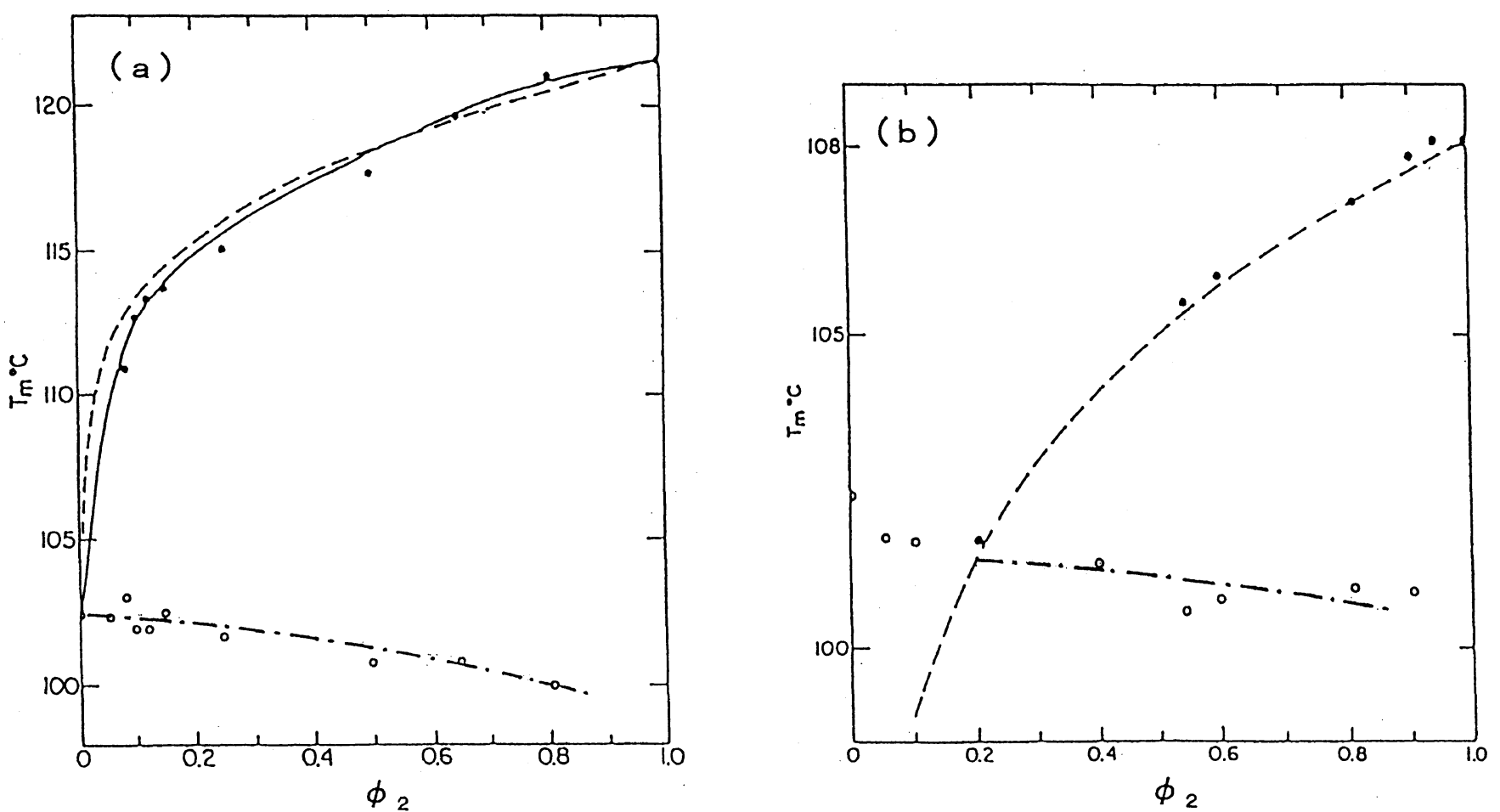

Figure 1. Melting temperature diagram for binary mixtures of (a) $\mathrm{PE} 2000$ and $\mathrm{C}_{60} \mathrm{H}_{122}$ and (b) $\mathrm{PE} 1000$ and $\mathrm{C}_{60} \mathrm{H}_{122}$ cited from literature data of Mandelkern et al. (ref 6). Chain curves, (a) $\lambda_{2}=0.25$ and (b) $\lambda_{2}=0.10$ are calculated from eq 18 .

crystalline substance as the component 1 . We assume that $T_{\mathrm{m} 2}>T_{\mathrm{m} 1}$. As the higher melting temperaturecomposition relation for the component 2 , we can employ eq 12 derived in the previous section. On the contrary, as the lower melting temperature-composition relation for the component 1 , we should not use eq 11 itself, but the following modification is needed.

Since the component 2 is a semicrystalline polymer, at the melting temperature of the component $1, T=T_{\mathrm{m} 1}$ $\left(\lambda_{1}=1\right)$, the chemical potential of unit of the component 1 in the surrounding liquid phase is influenced by the crystalline-amorphous proportion of the component 2 , i.e., $0<\lambda_{2}<1$. Then, by putting $\lambda_{1}=1$ and $\lambda_{2}=\lambda_{2}$ in eq 6 , the effective volume fraction of the component $2, \phi_{2}^{\prime}$ as the role of a diluent, is given by

$$
\phi_{2}^{\prime}=\frac{\phi_{2} \lambda_{2}}{\phi_{1}+\phi_{2} \lambda_{2}}=\frac{\phi_{2} \lambda_{2}}{1-\phi_{2}\left(1-\lambda_{2}\right)}
$$

By substituting $\phi_{2}^{\prime}$ of eq 17 for $\phi_{2}$ in eq 11 , we have

$$
\begin{aligned}
\frac{1}{T_{\mathrm{m} 1}}-\frac{1}{T_{\mathrm{m} 1}^{\mathrm{o}}}= & -\left(\frac{R}{\Delta H_{\mathrm{u}}}\right)\left\{\left(\frac{1}{x_{1}}\right) \ln \left[\frac{1-\phi_{2}}{1-\phi_{2}\left(1-\lambda_{2}\right)}\right]\right. \\
& \left.+\left(\frac{1}{x_{1}}-\frac{1}{x_{2}}\right)\left[\frac{\phi_{2} \lambda_{2}}{1-\phi_{2}\left(1-\lambda_{2}\right)}\right]\right\}
\end{aligned}
$$

This is the lower melting temperature-composition relation for the component 1 in the present system.

Mixtures of Polyethylene and n-Alkane. Investigations of melting temperature diagram for binary mixtures of polyethylene (PE) and normal alkane were reported by several researchers. ${ }^{6,9-11}$ Among them, we will concern with the work of Mandelkern et al. ${ }^{6}$ for the comparison of experimental data with the theoretical treatment derived in this paper. The analysis of higher melting temperature-composition relation was made in their article. Here we present an attempt to apply the lower melting temperature relationship of eq 18 to the experimental data of Mandelkern et al. ${ }^{6}$

For binary mixtures of PE2000 and $\mathrm{C}_{60} \mathrm{H}_{122}$ and those of PE1000 and $\mathrm{C}_{60} \mathrm{H}_{122}$, the calculated curves by using eq 18 with $\lambda_{2}=0.25$ and $\lambda_{2}=0.10$ are shown by chain curves in Figures 1(a) and 1(b), corresponding to Figures 3(b) and 4 of the original paper of Mandelkern et al., ${ }^{6}$ respectively. It seems that the calculated curves exhibit the decreasing trend of lower melting temperature in harmony with experimental data fairly well, although the quantitative agreement over the whole range of composition is insufficient.

\section{Mixutres of Two Semicrystalline Polymers}

Higher and Lower Melting Temperatures-Composition Relations. In this system, higher melting temperature component shall be designated as the component 2 , so we have $T_{\mathrm{m} 2}>T_{\mathrm{m} 1}$. The higher melting temperature relationship of $T_{\mathrm{m} 2}$ can be obtained from eq 10 by putting $T=T_{\mathrm{m} 2}, \lambda_{1}=1$, and $\lambda_{2}=1$

$$
\begin{aligned}
& \frac{1}{T_{\mathrm{m} 2}}-\frac{1}{T_{\mathrm{m} 2}^{\mathrm{o}}}=-\left(\frac{R}{\Delta H_{\mathrm{u} 2}}\right) \\
& \quad \times\left\{\frac{\ln \phi_{2}}{x_{2}}+\left[\frac{1}{x_{2}}-\left(\frac{z_{2}}{z_{1}}\right)\left(\frac{1}{x_{1}}\right)\right]\left(1-\phi_{2}\right)+z_{2} \chi\left(1-\phi_{2}\right)^{2}\right\}
\end{aligned}
$$

In case of $\left(z_{2} / z_{1}\right)=1$ and $\chi=0$, eq 19 is reduced to eq 12. It is to be noted that eq 19 is converted to a somewhat simpler expression by putting $z_{1}=1$ as follows 


$$
\begin{aligned}
& \frac{1}{T_{\mathrm{m} 2}}-\frac{1}{T_{\mathrm{m} 2}^{\mathrm{o}}}=-\left(\frac{R}{\Delta H_{\mathrm{u} 2}}\right)\left(\frac{V_{\mathrm{u} 2}}{V_{\mathrm{u} 1}}\right) \\
& \quad \times\left[\frac{\ln \phi_{2}}{m_{2}}+\left(\frac{1}{m_{2}}-\frac{1}{m_{1}}\right)\left(1-\phi_{2}\right)+\chi\left(1-\phi_{2}\right)^{2}\right]
\end{aligned}
$$

where $m_{1}\left(=z_{1} x_{1}\right)$ and $m_{2}\left(=z_{2} x_{2}\right)$ are the numbers of segments per molecule, $V_{\mathrm{u} 1}$ and $V_{\mathrm{u} 2}$ are the molar volumes of repeating units of the components 1 and 2, respectively, and $\left(z_{2} / z_{1}\right)=\left(V_{\mathrm{u} 2} / V_{\mathrm{u} 1}\right)$. Equation 20 is just the relationship derived by Nishi and Wang ${ }^{5}$ for binary mixtures for a crystalline-amorphous polymer pair. For large values of $x_{1}$ and $x_{2}$, eq 19 reduces to

$$
\frac{1}{T_{\mathrm{m} 2}}-\frac{1}{T_{\mathrm{m} 2}^{\mathrm{o}}}=-\left(\frac{R}{\Delta H_{\mathrm{u} 2}}\right) z_{2} \chi\left(1-\phi_{2}\right)^{2}
$$

As an alternative expression, if we employ the interaction energy density $B\left(\mathrm{cal} \mathrm{cm}^{-3}\right), z_{2} \chi$ can be rewritten as $B V_{\mathrm{n} 2} / R T_{\mathrm{m} 2}$ at $T=T_{\mathrm{m} 2}$.

At the lower melting temperature of the component $1, T=T_{\mathrm{m} 1}$, where $\lambda_{1}=1$, again we must consider the situation that the chemical potential of unit of the component 1 in the surrounding liquid phase is influenced by the crystalline-amorphous proportion of the component 2 , i.e., $0<\lambda_{2}<1$, since the component 2 is a semicrystalline polymer. Then the required lower melting temperature relationship of the component 1 at $T=T_{\mathrm{m} 1}$ can be obtained by substituting $\phi_{2}^{\prime}$ of eq 17 for $\phi_{2}$ in eq 9 .

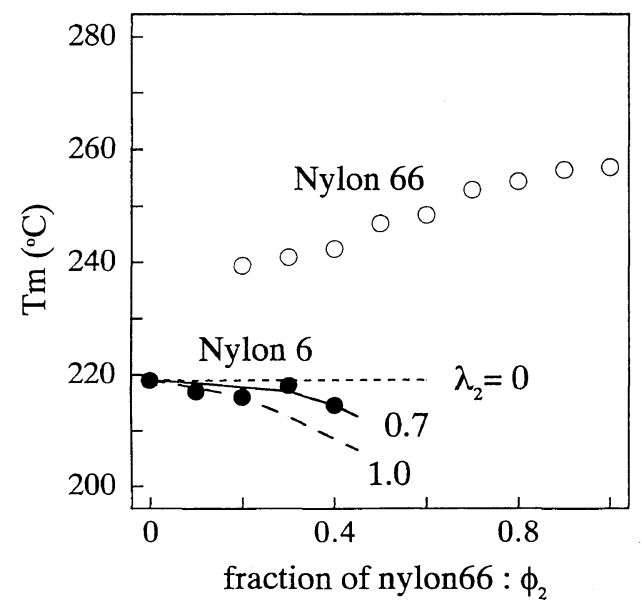

Figure 2. Melting temperature diagram for binary mixtures of nylon 6 and nylon 66 of our data (ref 12). Curves for $\lambda_{2}=0,0.7$, and 1.0 are culculated from eq 23

$$
\begin{aligned}
\frac{1}{T_{\mathrm{m} 1}}-\frac{1}{T_{\mathrm{m} 1}^{\mathrm{o}}}= & -\left(\frac{R}{\Delta H_{\mathrm{u} 1}}\right)\left\{\left(\frac{1}{x_{1}}\right) \ln \left[\frac{1-\phi_{2}}{1-\phi_{2}\left(1-\lambda_{2}\right)}\right]\right. \\
& +\left[\frac{1}{x_{1}}-\left(\frac{z_{1}}{z_{2}}\right)\left(\frac{1}{x_{2}}\right)\right]\left[\frac{\phi_{2} \lambda_{2}}{1-\phi_{2}\left(1-\lambda_{2}\right)}\right] \\
& \left.+z_{1} \chi\left[\frac{\phi_{2} \lambda_{2}}{1-\phi_{2}\left(1-\lambda_{2}\right)}\right]^{2}\right\}
\end{aligned}
$$

For large values of $x_{1}$ and $x_{2}$, eq 22 reduces to

$$
\frac{1}{T_{\mathrm{m} 1}}-\frac{1}{T_{\mathrm{m} 1}^{\mathrm{o}}}=-\left(\frac{R}{\Delta H_{\mathrm{u} 1}}\right) z_{1} \chi\left[\frac{\phi_{2} \lambda_{2}}{1-\phi_{2}\left(1-\lambda_{2}\right)}\right]^{2}
$$

As an alternative expression for the polymer-polymer interaction parameter, $z_{1} \chi$ can be rewritten as $B V_{\mathrm{u} 1} / R T_{\mathrm{m} 1}$ at $T=T_{\mathrm{m} 1}$.

Mixtures of Nylon 6 and Nylon 66. Figure 2 shows melting temperature diagram for binary mixtures of nylon 6 (the component 1) and nylon 66 (the component 2) obtained from measurements of differential scanning calorimetry (DSC) recently made in our laboratory. The experimental results and the analysis of higher melting temperature-composition relation of nylon 66 were reported in a previous paper. ${ }^{12}$

In this system, the formation of crystalline phase of nylon 66 predominates and in the range where the content of nylon 6 is small, a profile of nylon 66 overshadows that of nylon 6 , so the coexistence of two peaks of the two nylon components was observed in the range where $\phi_{2}=0.1-0.4$. Even in the narrow range of composition, we can recognize the slowly decreasing tendency of the lower melting temperature of nylon 6 as the content of nylon 66 increases. We attempted to apply eq 23 to experimental data of lower melting temperaturecomposition relation nylon 6 with the use of literature data of $\Delta H_{\mathrm{u} 1}=5100\left(\mathrm{cal} \mathrm{mol}^{-1}\right)^{13}$ and $B=-5.1$ (cal $\left.\mathrm{cm}^{-3}\right) .{ }^{12}$ The theoretical calculations are indicated in Figure 2; as can be seen, $\lambda_{2}=0.7$ is the best fit.

\section{DISCUSSION}

In Figures 3(a), 3(b), and 3(c), three types of phase diagram are schematically shown for the corresponding three categories of binary mixture system of two crystallizable components : (A) two wholly-crystalline substances, (B) combination of a semicrystalline polymer and a wholly crystalline substance, and (C) two semi-

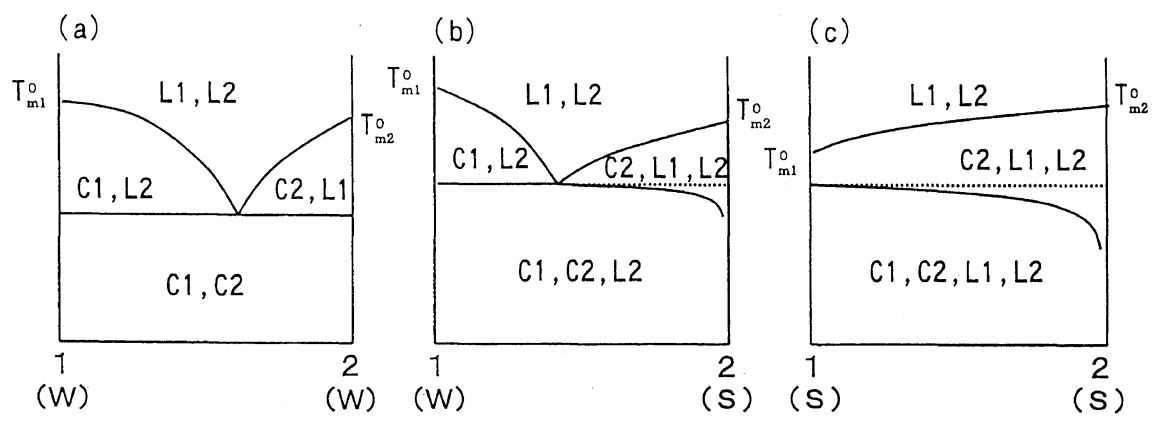

Figure 3. Schematic diagram for binary mixtures of two crystallizable components (components 1 and 2 ) of wholly-crystalline substances and semicrystalline polymers. Three categories: (a) two wholly-crystalline substances ((W)1 and (W)2), (b) a wholly-crystalline substance and a semicrystalline polymer ((W)1 and (S)2), and (c) two semicrystalline polymers $((\mathrm{S}) 1$ and (S)2). Symbols C and L designate the crystalline and the liquid phases, respectively. 
crystalline polymers. In the category A, we know numerous examples of binary mixtures whose components are metals and organic crystalline substances of low molecular weight. The principle of the phase behavior of the category A was established in classical thermodynamics, that is, higher melting temperatures of two components decrease and intersect at the eutectic point, while the lower melting temperature determined at the eutectic point is kept to be constant irrespective of composition as a consequence of the Gibbs phase rule.

This principle is violated when a semicrystalline polymer is introduced in the system as a higher melting temperature component (the component 2). In this case, the lower melting temperature component (the component 1), either a semecrystalline polymer, or a whollycrystalline substance, decreases as the content of the component 2 increases, depending on the crystallineamorphous proportion of the component 2 in the surrounding region of crystalline phase of the component 1. Thus, thermodynamic properties of the surrounding region are not determined uniquely by the composition. This fact causes the failure of the Gibbs phase rule in the present binary system. It is to be emphasized that the present work is concerned with systems beyond the scope of those in truely equilibrium state as treated in classical thermodynamics.

The analysis of the decreasing tendency of lower melting temperature $T_{\mathrm{m} 1}$ as a function of composition enables us to estimate the amorphous fraction of the component 2, i.e., $\lambda_{2}$. The physical meaning of $\lambda_{2}$ thus obtained may be regarded as a parameter representing the degree of diluent effect, which shall be called as "the activity coefficient of diluent," caused by semicrystalline polymer of the component 2 . It is interesting to compare the obtained value of $\left(1-\lambda_{2}\right)$ with the degree of crystallinity evaluated independently from thermal, structural or morphological basis. We believe that the study of lower melting temperature-composition relation in binary mixtures of the categories $\mathrm{B}$ and $\mathrm{C}$ will provide significant information concerning the crystalline-amorphous phase transformation in semicrystalline polymer systems, although no interest has been directed to this problem so far.

Acknowledgments. This work was supported by the international joint research grant of NEDO (New Energy and Industrial Technology Development Organization). We wish to thank Dr. A. Toda for his interest and advice in the course of this work.

\section{REFERENCES}

1. P. J. Flory, J. Chem. Phys., 17, 223 (1949).

2. P. J. Flory, "Principles of Polymer Chemistry," Cornell University, Ithaca, N.Y., 1953, Chapter XIII-2.

3. L. Mandelkern, "Crystallization of Polymers," McGraw-Hill, New York, N.Y., 1963.

4. L. Mandelkern, in "Comprehensive Polymer Science Vol. 2, Polymer Properties,' C. Booth and C. Price, Ed., Pergamon Press, New York, N.Y., 1989, Chapter 11.

5. T. Nishi and T. T. Wang, Macromolecules, 8, 909 (1975).

6. L. Mandelkern, F. L. Smith, and E. K. Chan, Macromolecules, 22, 2663 (1989).

7. Reference 2. Chapter XIII-1c.

8. P. J. Flory, Faraday Discuss. Chem. Soc., 49, 7 (1970).

9. P. Smith and R. St. John Manley, Maclomolecules, 12, 483 (1979).

10. C. Nakafuku, M. Yasuniwa, and S Tsubakihara, Polym. J., 22, $110(1990)$

11. C. Nakafuku, H. Nakagawa, M. Yasuniwa, and S. Tsubakihara, Polymer, 32, 696 (1991)

12. T. Matsuda , T. Shimomura, and M. Hirami, Polym. J., 31, 795 (1999).

13. Reference 3. Chapter 5-2, p 120. 